\title{
Aplicação forense do GPR na localização inumações na área da Guerrilha do Araguaia
} Lucas Barros de Andrade $\star^{1}$ - lucas.lba@dpf.gov.br

Eduardo Xavier Seimentz ${ }^{2}$, Gustavo Ferraz de Oliveira ${ }^{1}$, Marcelo de Lawrence Bassay Blum ${ }^{1}$, Marcio Maciel Cavalcanti ${ }^{2}$, Péricles de Brito Macedo ${ }^{2}$, Welitom Rodrigues Borges ${ }^{2}$

${ }^{1}$ Departamento de Polícia Federal, ${ }^{2}$ Universidade de Brasília

Copyright 2012, SBGf - Sociedade Brasileira de Geofísica

Este texto foi preparado para a apresentação no V Simpósio Brasileiro de Geofísica Salvador, 27 a 29 de novembro de 2012. Seu conteúdo foi revisado pelo Comitê Técnico do V SimBGf, mas não necessariamente representa a opinião da SBGf ou de seus associados. É proibida a reprodução total ou parcial deste material para propósitos comerciais sem prévia autorização da SBGf.

\section{Abstract}

As part of the searches for members of the Araguaia Guerilla (1972-1974) remains, one of the activities conducted by the Araguaia Working Group, surveys using Ground Penetrating Radar (GPR) were carried out in two areas in order to indicate burial sites. The areas, measuring $5 \times 5 \mathrm{~m}$, were investigated using an antenna with central frequency in $400 \mathrm{Mhz}$ and lines spaced of $0,1 \mathrm{~m}$. The interpretation of the 3D data indicated four possible targets. The excavations have shown that three of the targets were buried human remains and the other one was associated to animals burrows.

\section{Introdução}

O presente trabalho faz parte das atividades do Grupo de Trabalho do Araguaia (GTA), que tem entre seus objetivos a localização de restos mortais de participantes do movimento da Guerrilha do Araguaia, entre 1972 e 1974 (Campos Filho, 2012).

Visando à localização de possíveis inumações em região conhecida por Fazenda das Abóboras, na zona rural do Município de São Geraldo do Araguaia - PA (Figura 1), foram realizados levantamentos de radar de penetração no solo (GPR, do inglês Ground Prenetrating Radar). A interpretação dos dados de GPR permitiu a indicação de alvos para escavação. A metodologia empregada e os resultados obtidos são apresentados neste resumo.

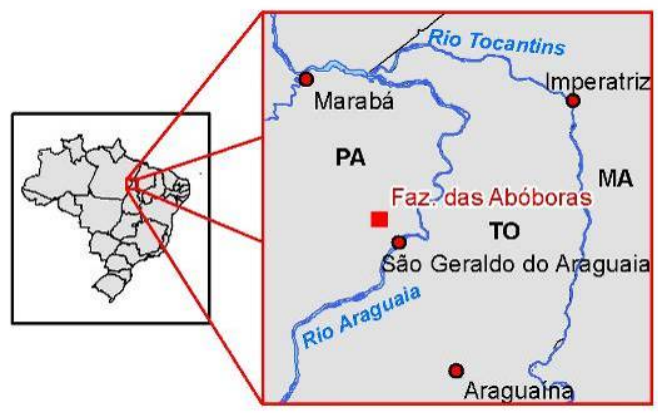

Figura 1 - Localização da área conhecida por Fazenda das Abóboras, na zona rural do Município de São Geraldo do Araguaia - PA.

\section{Metodologia}

O GPR é um método geofísico eletromagnético que se utiliza de ondas de rádio de alta frequência para estudos de estruturas subsuperficiais (Anann, 1992). O equipamento dispõe de duas antenas, uma antena transmissora emite um pulso para o subsolo, onde este pulso é refletido, refratado e difratado para uma antena receptora. Este sinal recebido é digitalizado, sendo possível a visualização de padrões de reflexão relacionados a objetos no subsolo.

A utilização do GPR para investigações forenses visando a localização de restos mortais encontra-se bem documentada na literatura (Strongman, 1992; Isaacson, et al. 1999; Hammon III, et al. 2000; Nascimento, 2009).

A área examinada foi indicada como local de inumações de restos mortais de participantes da Guerrilha do Araguaia por colaborador do GTA. No local, foram observados restos de cruzes de madeira, vestígios interpretados como indicadores da existência de sepulturas no local.

Baseando-se nas indicações do colaborador do GTA, foram selecionadas 2 (duas) áreas de $5 \times 5 \mathrm{~m}$, designadas por Área 1 e Área 2, onde foram realizados os levantamentos de GPR (Figura 2).

A aquisição de dados de GPR em ambas as áreas foi realizada em linhas espaçadas de $0,1 \mathrm{~m}$, de SW para NE na Área 1 e NNE para SSW na Área 2, sendo adquiridas 51 seções em cada área.

O equipamento GPR utilizado foi o SIR3000, fabricado pela empresa Geophysical Survey Systems - GSSI e de propriedade do Departamento de Polícia Federal, acoplado a uma antena blindada de com freqüência central em 400MHz (Figura 3). Os parâmetros de campo utilizados foram: espaçamento entre os traços de $0,02 \mathrm{~m}$, 1024 amostras por traço, janela temporal de 60ns e frequência de amostragem de $8500 \mathrm{MHz}$. Os traços foram registrados continuamente com o auxílio de uma roda odométrica. 


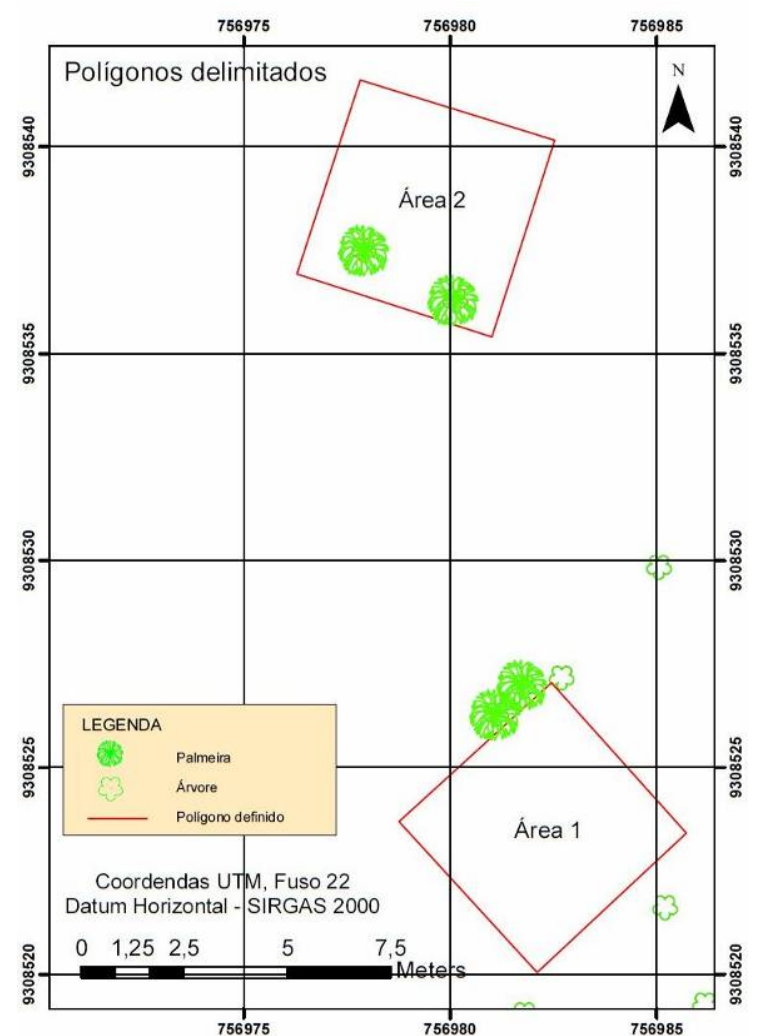

Figura 2 - Planta de situação das áreas onde foram realizados os levantamentos de GPR. (Figura elaborada pela Equipe de Topografia do GTA).

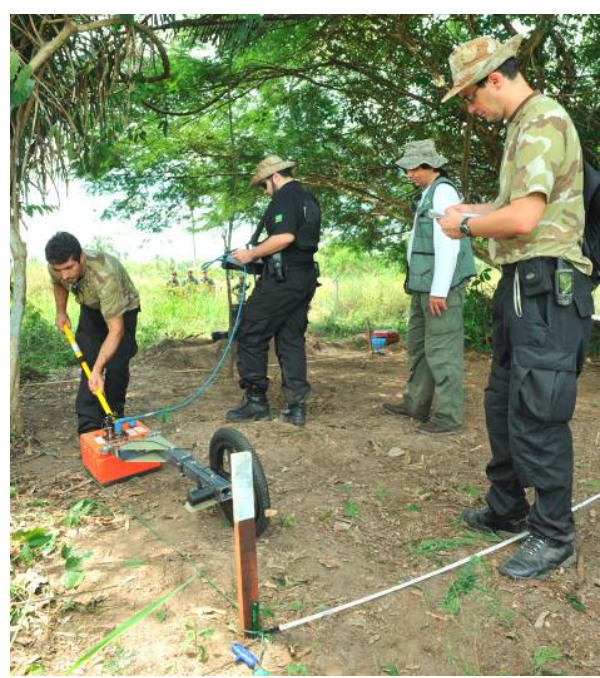

Figura 3 - Levantamento de GPR utilizando o equipamento SIR3000, fabricado pela empresa GSSI, acoplado a uma antena blindada de com freqüência central em $400 \mathrm{MHz}$.
Os dados foram processados no software ReflexW, versão 6.0 (Sandmeier, 2011). A rotina de processamento consistiu em conversão de formato do arquivo, ajuste do tempo zero, filtros temporal (dewow e passa-banda), ganho (decaimento de energia), migração no domínio do tempo (fk migration - Stolt) e conversão em profundidade (velocidade constante).

Para a obtenção da velocidade eletromagnética no meio, foi enterrada peça metálica com $0,05 \mathrm{~m}$ de diâmetro a $0,63 \mathrm{~m}$ de profundidade fora da área. Sobre esse artefato, foi efetuado um pequeno perfil de GPR (Figura 4). Conhecendo-se a profundidade real do mesmo, bem como o evento hiperbólico relacionado à picareta, obtevese a velocidade média de propagação da onda eletromagnética no meio de $0,128 \mathrm{~m} / \mathrm{ns}$, utilizada na inversão dos dados. Com a janela temporal de 60ns foi possível investigar até a profundidade aproximada de $3,5 \mathrm{~m}$.

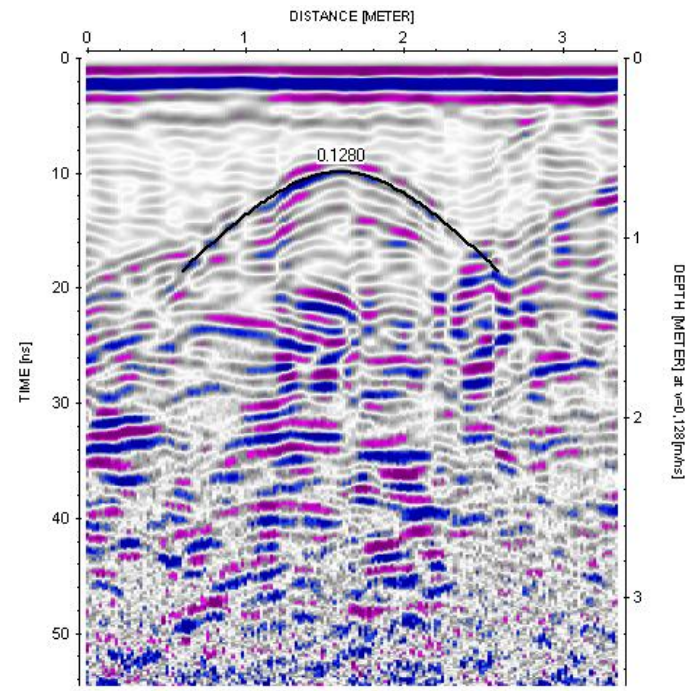

Figura 4 - Perfil de GPR executado sobre peça metálica para determinação da velocidade eletromagnética do meio. Considerando a profundidade $0,63 \mathrm{~m}$ e o tempo de resposta de 9,84ns, foi obtida velocidade de $0,128 \mathrm{~m} / \mathrm{ns}$.

Além do processamento bidimensional, o conjunto de dados foi interpolado tridimensionalmente, fornecendo modelos 3D das áreas investigadas.

\section{Resultados}

Os resultados de GPR foram analisados através de seções 2D individuais, bem como através de cortes em profundidade efetuados no modelo tridimensional.

Após análises, foram indicadas 4 anomalias com padrão similar a inumações: os Alvo 1.1 em profundidade estimada de $1,25 \mathrm{~m}$, Alvo $1.2 \mathrm{em}$ profundidade estimada de $1,2 \mathrm{~m}$ e Alvo 1.3 em profundidade estimada de $0,85 \mathrm{~m}$ na Área 1 (Figuras 5 e 6) e Alvo 2.1 em profundidade estimada de $1,1 \mathrm{~m}$ na Área 2 (Figura 7). 


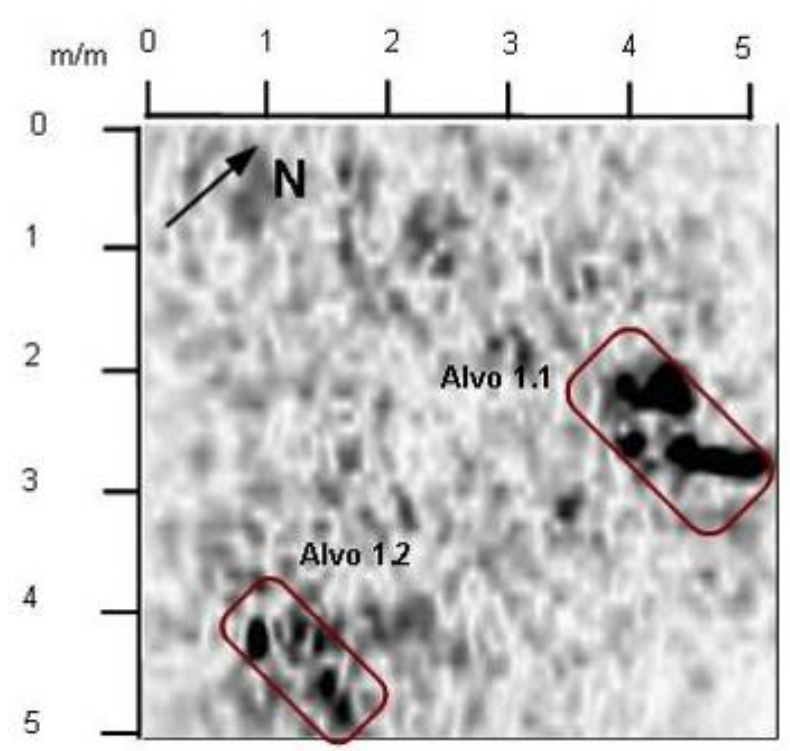

Figura 5 - Corte horizontal no modelo tridimensional da Área 1 em profundidade aproximada de 1,2m, com indicações dos Alvos 1.1 e 1.2.

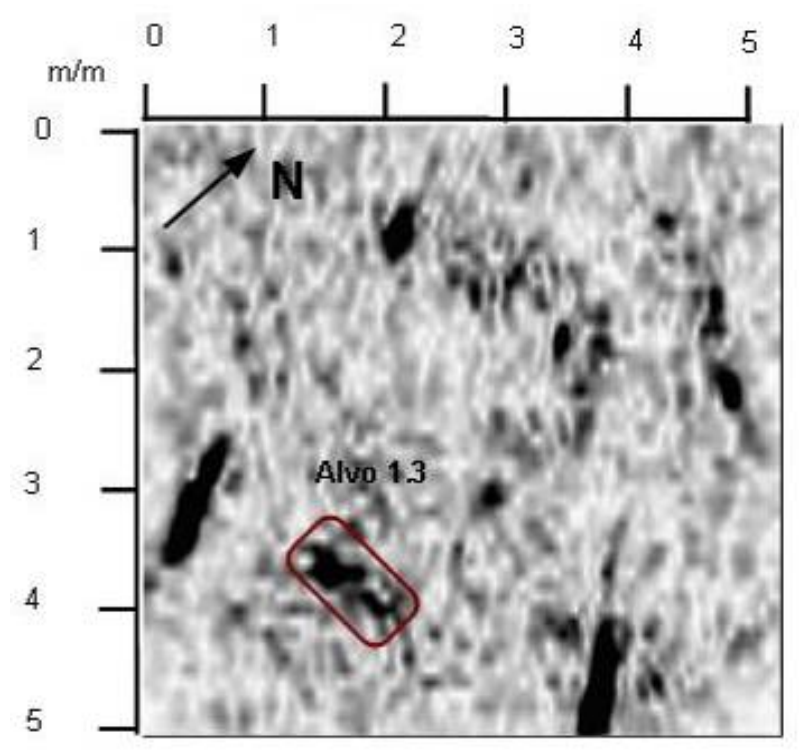

Figura 6 - Corte horizontal no modelo tridimensional da Área 1 em profundidade aproximada de 0,9m, com indicação do Alvo 1.3.

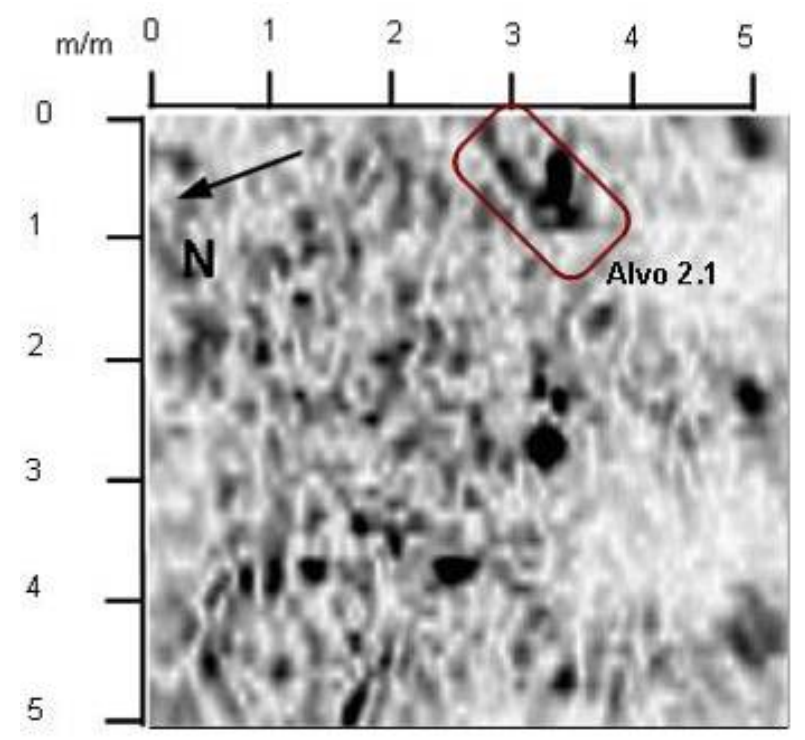

Figura 7 - Corte horizontal no modelo tridimensional da Área 2 em profundidade aproximada de 1,1m, com indicação do Alvo 2.1.

As anomalias indicadas foram investigadas por meio de escavações coordenadas pela Equipe de Antropologia Forense do GTA.

Durante as escavações, verificou-se que o Alvo 1.1 estava relacionado a redes de túneis de animais (Figura 8) e os Alvos 1.2 e 1.3 eram realmente inumações (Figuras 9 e 10). O Alvo 2.1 também se tratava de inumação (Figura 11).

Foi encontrada ainda inumação não indicada pelo levantamento geofísico (Figura 12).

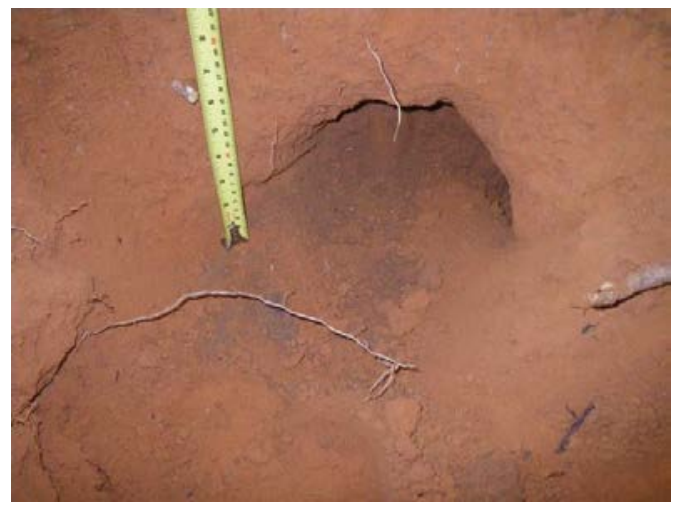

Figura 8 - Cavitação parte da rede de túneis de animais associada ao Alvo 1.1. 


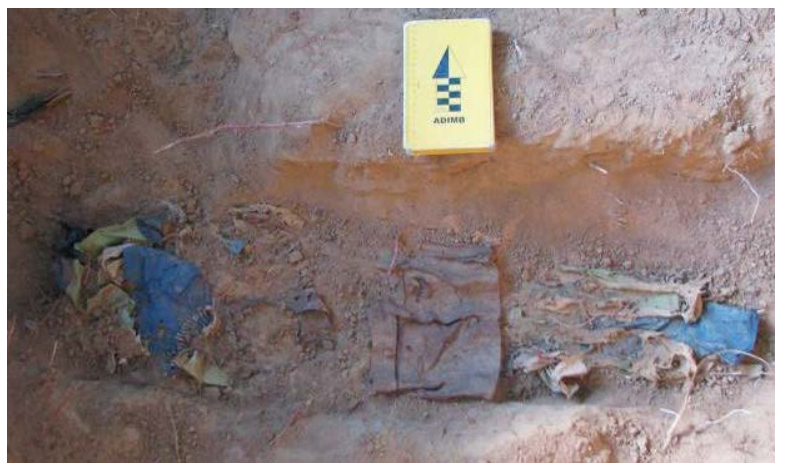

Figura 9 - Inumação localizada pelo levantamento de GPR na Área 1 (Alvo 1.2).

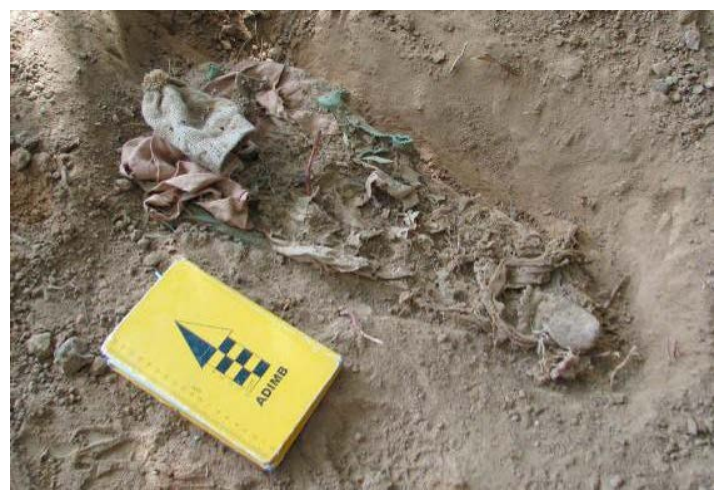

Figura 10 - Inumação localizada pelo levantamento de GPR na Área 1 ( Alvo 1.3)

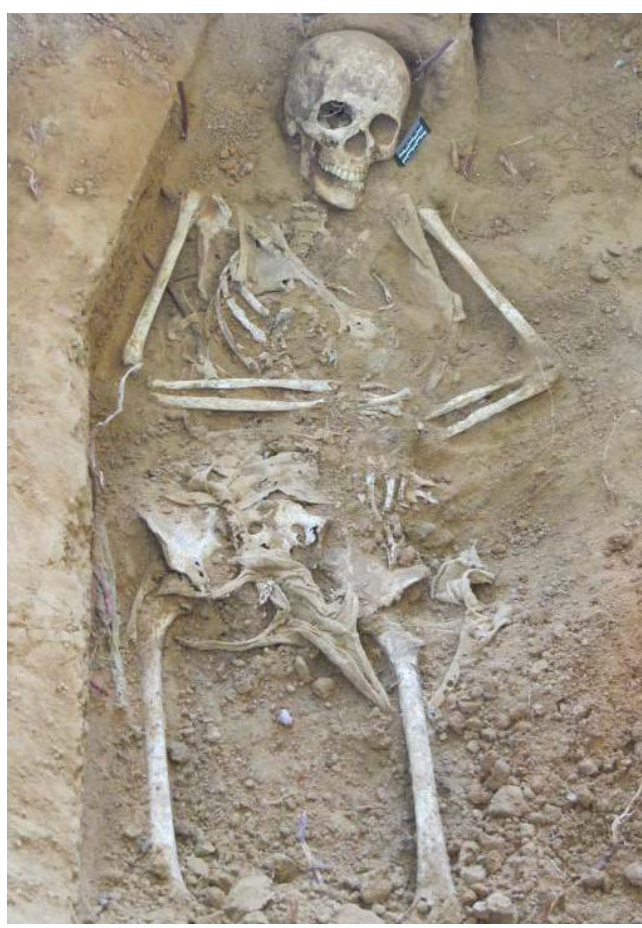

Figura 11 - Inumação localizada pelo levantamento de GPR na Área 2 (Alvo 2.1).

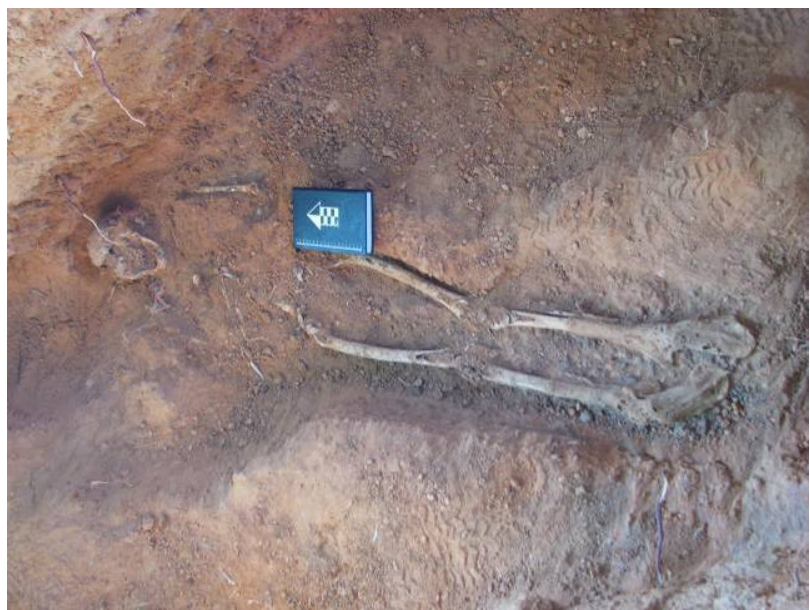

Figura 12 - Inumação não identificada pelo levantamento de GPR, sendo observada em análise posterior.

\section{Discussão e Conclusões}

A análise dos perfis de GPR e do modelo tridimensional nas áreas investigadas permitiu identificar anomalias relacionadas a inumações, ainda que um dos alvos indicados, Alvo 1.1, se tratasse de cavitações, relacionadas a rede de túneis de animais, com respostas de alta intensidade.

Em geral, a alteração na estrutura do solo decorrente das escavações é o que efetivamente é detectado pelo método geofísico, e não as inumações em si.

No caso dos Alvos 1.2 e 1.3, havia considerável quantidade de tecido sintético preservada, o que pode ter configurado um forte refletor resultando em um sinal de resposta mais intenso, haja vista que foi encontrada outra inumação na Área 1 não detectada pelo levantamento de GPR.

Acima e ao redor da inumação que não foi identificada pelos levantamentos de GPR, havia grande quantidade de cavitações relacionadas a túneis de animais, cuja resposta de alta intensidade do sinal possivelmente obliterou a anomalia relacionada a essa inumação.

\section{Agradecimentos}

Agradecimentos a todos os integrantes do Grupo de Trabalho do Araguaia que colaboraram na realização deste trabalho.

\section{Referências}

Annan, A.P, 1992. Ground Penetrating Radar, Workshop Notes. Sensores e Software, Inc. Internal Report. pp.130.

Campos Filho,R.P., 2012. Guerrilha do Araguaia - a esquerda em armas ( $2^{a}$ edição, atualizada e ampliada). Fundação Maurício Grabois / Editora Anita garibaldi. 344 p. 
Hammon III, W. S., Mcmechan, G. A., Zeng, X., 2000. Forensic GPR: finite-difference simulations of responses from buried human remains. Journal of Applied Geophysics, Vol. 45: $171-186$.

Isaacson, J.R., Hollinger, E., Gundrum, D., Baird, J., 1999. A Controlled Archaeological Test Site Facility in Illinois: Training and Research in Archaeogeophysics. Journal of Field Archaeology, Vol. 26(2): 227-236.

Nascimento, W.G. 2009. Investigação geofísica ambiental e forense nos cemitérios do Bengui e do Tapanã (BelémPA). Dissertação de Mestrado, Universidade Federal do Pará, Brasil, 150p.

Sandmeier, K.J., 2011. Sandmeier Software, Alemanha.

Strongman, K. B., 1992. Forensic applications of ground penetrating radar. In: Ground Penetrating Radar, ed. J. Pilon, Geological Survey of Canada, Paper 90-4, p. 203211. 